\title{
Innovationen im Coaching
}

\author{
Astrid Schreyögg
}

Seit der Jahrtausendwende hat sich das Interesse an Coaching geradezu sprunghaft verstärkt. Fillery-Travis \& Lane (2006) merkten schon 2006 an, dass es sich zu einem zwei Milliarden-Dollar-Markt in allen Industrienationen ausgewachsen hat. Und der Australier Grant konnte 2006 zeigen, dass auch die Publikationen im akademischen Bereich von 2001 bis 2005 gegenüber 1996 bis 2000 um266\% angestiegen sind (zit. nach Segers et al. 2011). Wir haben es hier also bei diesem Format mit turbulenten Entwicklungen in Theorie und Praxis zu tun. Praktiker befruchten die Theorie, und Theoretiker liefern der Praxis neue Anregungen. Im Verlauf dieser Prozesse wurde auch eine enorme Innovationskraft sichtbar. Das vorliegende Heft will einige dieser Entwicklungen einfangen.

Im ersten Beitrag berichten Harald Geißler, Melanie Hasenbein und Robert Wegener von E-Coaching. Die Autoren, die sich schon längere Zeit mit dem Thema beschäftigen, präsentieren ein Untersuchungsdesign mit ersten Ergebnissen. Dabei geht es um Telefon-Coaching in Verbindung mit einem Internetprogramm, das Erkenntnisse über spezifische Wirkfaktoren im Coaching nutzt. Das Format wirkte zwar auf viele Klienten zunächst sehr befremdlich, nach einigen positiven Erfahrungen haben sie ihre Skepsis jedoch überwunden.

Im zweiten Beitrag beschäftigen sich Stephan Daniel Richter und Maureen Marchioro mit der Affinität zur Ethik und zu Menschenbildern im Coaching anhand der Internetpräsenz von ausgewählten Coaching-Anbietern und -Ausbildern. Die Ergebnisse waren ernüchternd. Die Autoren legen daraufhin eine Checkliste vor, mit deren Hilfe die diesbezügliche Einordnung von Anbietern und Ausbildern für Interessenten möglich werden soll.

Gerhard Liska stellt in seiner Arbeit eine entwicklungspsychologische Perspektive für die beruflichen Beratungsformate Supervision, Coaching und Organisationsberatung vor. Dabei versucht der Autor zwei neue entwicklungspsychologische Ansätze für diese Beratungsformate nutzbar zu machen. Er beschreibt dann auch die entsprechenden Rahmenbedingungen und acht Ansatzpunkte für ein konzeptkonformes Lernen.

Online publiziert: 11.04 .2013

(C) Springer Fachmedien Wiesbaden 2013

Dr. A. Schreyögg $(\bowtie)$

Breisgauer Str. 29, 14129 Berlin, Deutschland

E-Mail: info@schreyoegg.de 
Von den vier Praxisberichten beschäftigt sich der erste mit dem Coaching von Führungskräften in Expertenorganisationen. Sybille Wölfing Kast erläutert die Besonderheit dieses Typs von Führungskräften anhand einiger Fallfacetten, um dann Schlüsselkonzepte fürs Coaching abzuleiten. Der zweite Praxisbericht von Claudia Guldenschuh, Psychologin in einer großen Bundesbehörde, ist ein Plädoyer für Life-Coaching, um auch in diesem Kontext eine gute Work-Life-Balance zu befördern. Simone Kurz, Senior Consultant bei Kienbaum, zeigt, wie Coaching zu einem festen Bestandteil systematischer und (strategischer) Personalentwicklung in einem System der öffentlichen Verwaltung werden konnte. Schließlich beschäftigt sich Silke Dinius mit einer Problematik, mit der Teams der Sozialen Arbeit konfrontiert sein können, wenn sie sich in ausweglos erscheinenden Situationen befinden. Sie zeigt anhand von Fallbeispielen auf, in welcher Weise Supervision helfen kann, Ohnmachtsgefühle zu überwinden, und erörtert damit verbundene ethische, pragmatische und methodische Fragestellungen.

Im Diskurs problematisiere ich, Astrid Schreyögg, die Entscheidung der DGSv, Supervision und Coaching gleichzusetzen. Diese Entscheidung scheint mir nicht nur aus fachlichen Gründen z.B. aufgrund der unterschiedlichen Ursprünge unsinnig, sondern auch aufgrund der unterschiedlichen Marktlage grob vereinfachend.

\section{Literatur}

Fillery-Travis, A. F., \& Lane, D. (2006). Does coaching work or are we asking the wrong question? International Coaching Psychology Review, 1, 23-36.

Segers, J., Vloberghs, D., \& Henderickx, E. (2011). Structuring an understanding the coaching industry: the coaching cube. Academy of Management, Learning/Education, 10(2), 204-222. 